\title{
Epidemiology of Nontuberculous Mycobacterial Infection, South Korea, 2007-2016
}

\author{
Hyewon Lee, ${ }^{1}$ Woojae Myung, ${ }^{1}$ Won-Jung Koh, Seong Mi Moon, Byung Woo Jhun
}

The prevalence and incidence of nontuberculous mycobacterial (NTM) infections increased in South Korea from 2007 to 2016. Annual prevalence of NTM infection increased to 39.6 cases $/ 100,000$ population in 2016 and annual incidence to 19.0 cases $/ 100,000$ population. Overall prevalence for the study period was higher in the elderly, in females, and in cities.

$\mathrm{T}$ he prevalence and incidence of nontuberculous mycobacterial (NTM) infection are increasing worldwide (1), and it is important to characterize the distribution of NTM infection by demographics or ecologic region to optimize disease control. However, data vary widely by study, and the epidemiology of NTM infection differs among countries. Although the number of patients with NTM infection has increased in South Korea (2), representative nationwide population-based epidemiologic data on the extent and distribution of NTM infection are lacking. We evaluated the prevalence, incidence, and spatial distribution of NTM infection in South Korea over a 10-year period, using national health insurance data.

\section{The Study}

We based our study on the Health Insurance Review and Assessment Service database, which includes universal health insurance claim data in South Korea. Approximately $97 \%$ of the population ( $\approx 52$ million persons) is included in the system (3). We obtained demographic and medical data from the database for 2007-2016. We defined NTM infection as the presence of a diagnostic code associated with NTM infection (International Classification of Diseases, 10th ed., code A31) as either a primary or secondary diagnosis (4) and claims data for acid-fast bacilli smears or mycobacterial culture.

We defined annual prevalence as the number of all patients with NTM infection alive during each year and annual incidence as the number of newly diagnosed NTM

Author affiliations: Seoul National University, Seoul, South Korea

(H. Lee); Seoul National University Bundang Hospital, Seoul

(H. Lee, W. Myung); Sungkyunkwan University School of

Medicine, Seoul (W.-J. Koh, S.M. Moon, B.W. Jhun) infections occurring each year after excluding patients who were diagnosed in a previous study period (e.g., patients diagnosed during 2007-2015 were excluded from the estimate of the 2016 incidence rate). We first calculated the crude prevalence rate and incidence rate using each year's total population as the denominator. To adjust for different population structures between years, we calculated the age- and sex-standardized annual prevalence rate and incidence rate of NTM disease using the 2005 population as a standard population, based on the Statistics Korea method (http://kostat.go.kr/portal/eng/index. action). First, we calculated the prevalence and incidence rates for age and sex groups by stratifying all NTM cases into the groups and dividing by the group-specific populations in each year. Then, we multiplied each year's group-specific rates and the group-specific populations in 2005 , combined the multiplied values, and divided by the 2005 total population. We calculated standardized overall period prevalence of NTM infection by age, sex, and administrative divisions using the population in the middle of the study period. We conducted statistical analyses using SAS version 9.4 (SAS Institute, http://www.sas.com). Because we used deidentified data in the study, institutional review board approval and patient consent were not required.

A total of 33,974 cases of NTM infection were identified during 2007-2016. The age- and sex-adjusted annual prevalence of NTM infection increased by more than 5-fold, from 6.7 cases/100,000 population in 2007 to 39.6 cases/100,000 population in 2016 (Figure 1). The age- and sex-adjusted annual incidence of NTM infection more than tripled, from 6.0 cases/100,000 population/year in 2008 to 19.0 cases/100,000 population/year in 2016. The overall period prevalence of NTM infection increased with patient age (Figure 2, panel A): prevalence was lowest at $\leq 19$ years of age (1.7 cases/100,000 population), increased dramatically after 50 years of age, and peaked in those $\geq 70$ years of age ( 223.0 cases $/ 100,000$ population). The overall period prevalence of NTM infection was higher among females in all age groups, except for those $\geq 70$ years of age (Figure 2, panel B).

In South Korea, the overall period prevalence of NTM infection tended to be higher in metropolitan cities, which

${ }^{1}$ These authors contributed equally to this article. 


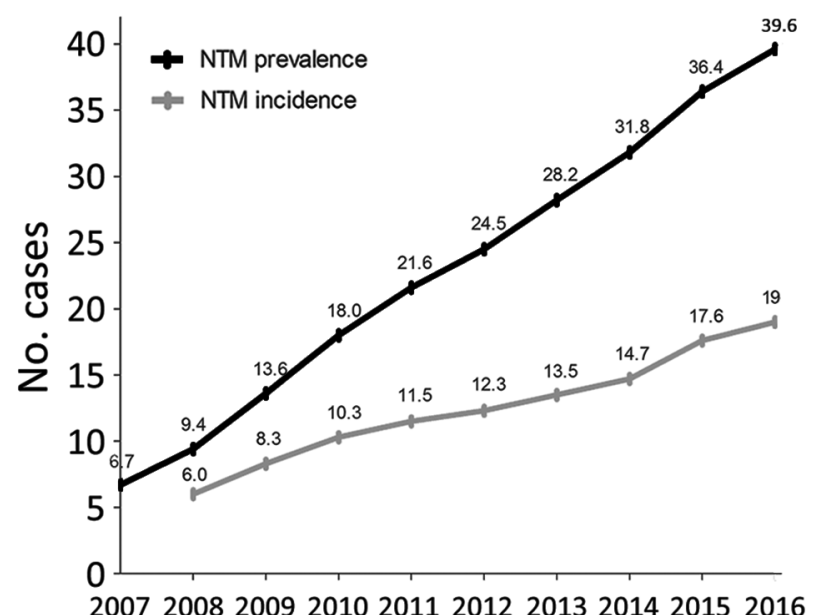

Figure 1. Annual prevalence (total no. cases/100,000 population) and incidence (no. new cases/100,000/year) of nontuberculous mycobacterial infection, adjusted for age and sex, South Korea, 2007-2016.

had greater population densities and higher income levels, than in the provinces (Table 1). The overall period prevalence exceeded 100 cases/100,000 population in most metropolitan cities $(5 / 7,71.4 \%)$ but did not exceed that rate in most provinces $(7 / 9,77.8 \%)$. The prevalence was highest in Gwangju and in Seoul, the capital of South Korea, both of which had $>250$ cases $/ 100,000$ population. Prevalence was lowest in Cheju, the largest and southernmost island (35.8 cases/100,000 population) (Appendix Figure, https:// wwwnc.cdc.gov/eid/article/25/3/18-1597-techapp1.pdf).

Analysis of age and underlying conditions indicated that most case-patients $(83.4 \%)$ were $>50$ years of age (Table $2)$. The most frequent underlying respiratory condition was asthma $(53.0 \%)$, followed by bronchiectasis $(43.6 \%)$ and chronic obstructive pulmonary disease (COPD) (32.3\%). Lung cancer was the most common malignancy (6.3\%). Depressive disorder was noted in $23.1 \%$ of case-patients.

\section{Conclusions}

We identified a remarkable increase in the prevalence and incidence of NTM infection in South Korea from 2007 to 2016. The overall period prevalence increased with patient age and was higher in female patients in most age groups. These trends are consistent with previous studies from Europe (4), the United States $(5,6)$, and Japan $(7,8)$. A representative US study that analyzed Medicare beneficiaries during 1997-2007 revealed that the prevalence of NTM disease increased from 20 to 47 cases/100,000 population and the prevalence was higher among women (4). A study conducted in Germany reported an increasing prevalence of NTM disease, from 2.3 cases/100,000 population in 2009 to 3.3 cases/100,000 population in 2014 (4). Studies in Japan, which is geographically close to South Korea, showed similar trends, but the rates were higher than those in the United States or Europe. The estimated annual prevalence of NTM disease in Japan in 2005 was 33-65 cases/100,000 population (7), and the incidence in 2015 was 14.7 cases $/ 100,000$ population ( 8 ), which were similar to our data. These results suggest that NTM infection is increasing globally, especially in East Asia, and that epidemiologic distribution can be affected by region or ethnicity. In particular, some studies have indicated that people in East Asia are vulnerable to NTM infection $(9,10)$.

Another notable finding in our study was the difference in NTM prevalence by administrative division, which indicates that NTM infection is related to population density and the degree of urbanization. Supporting our data, other studies have attributed NTM infection to environmental exposure (9), and recent studies in the United States revealed that areas with high risk for NTM infection had higher population densities or higher education and income levels $(9,11)$. In South Korea, the population density is highest in metropolitan cities. These data suggest the importance of epidemiologic surveillance in understanding NTM infection.
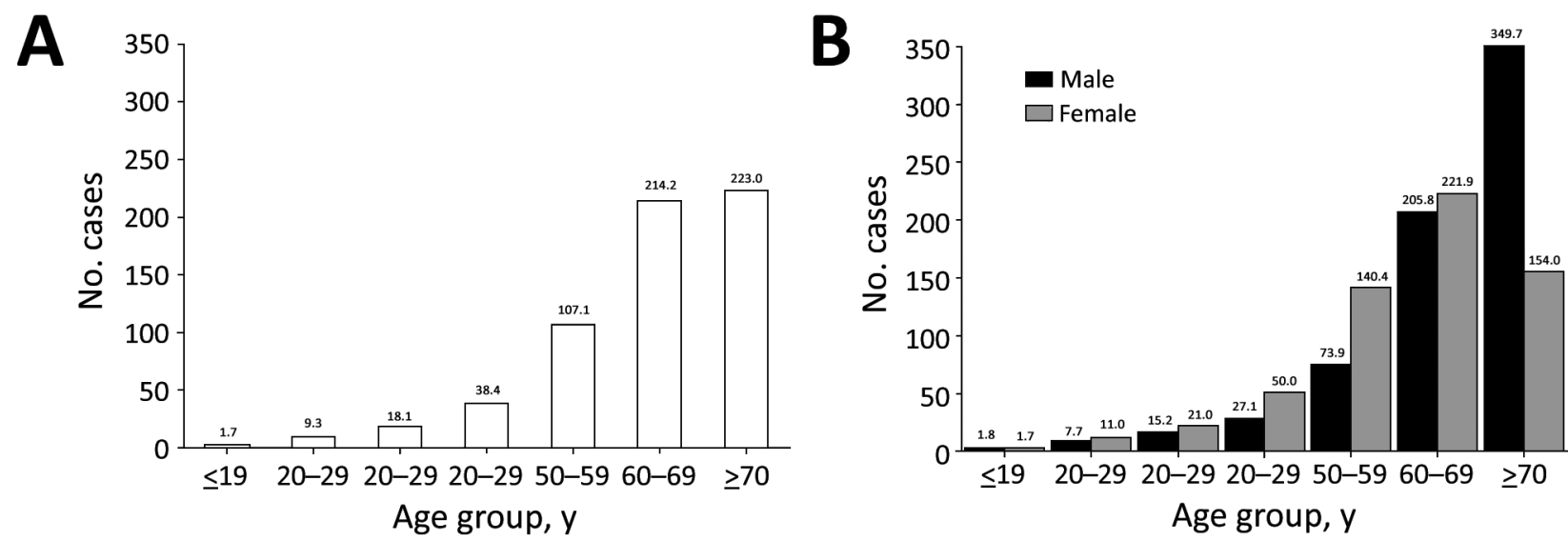

Figure 2. Overall period prevalence (total no. cases/100,000 population) of nontuberculous mycobacterial infection, by age group (A) and by age group and sex (B), South Korea, 2007-2016. 
We frequently observed airway diseases in NTM infection in our study. A US study also reported COPD in $41 \%$ and bronchiectasis in $37 \%$ of NTM patients (10),

\begin{tabular}{|c|c|c|}
\hline District & $\begin{array}{c}\text { No. } \\
\text { infections }\end{array}$ & $\begin{array}{c}\text { No. cases } / 100,000 \\
\text { population }\end{array}$ \\
\hline \multicolumn{3}{|l|}{ Metropolitan city } \\
\hline Seoul & 13,671 & 254.5 \\
\hline Busan & 2,055 & 102.6 \\
\hline Daejeon & 1,384 & 194.4 \\
\hline Gwangju & 2,121 & 302.0 \\
\hline Daegu & 1,642 & 119.9 \\
\hline Incheon & 1,169 & 86.3 \\
\hline Ulsan & 401 & 81.5 \\
\hline \multicolumn{3}{|l|}{ Province } \\
\hline Gyeonggi-do & 6,195 & 110.2 \\
\hline Gangwon-do & 1,107 & 108.9 \\
\hline Jeollabuk-do & 1,213 & 98.4 \\
\hline Jeollanam-do & 470 & 35.1 \\
\hline Gyeongsangbuk-do & 497 & 26.8 \\
\hline Gyeongsangnam-do & 1,201 & 66.0 \\
\hline Chungcheongbuk-do & 285 & 30.6 \\
\hline Chungcheongnam-do & 444 & 35.2 \\
\hline Cheiu-do (island) & 119 & 35.8 \\
\hline
\end{tabular}

Table 2. Sex, age, and underlying conditions among patients with nontuberculous mycobacterial infection, South Korea, 2007-2016*

\begin{tabular}{lc}
\hline Category & No. $(\%)$ infections, \\
$\mathrm{n}=33,974$
\end{tabular}

whereas a study in Germany noted COPD or emphysema in $62 \%-79 \%$ of patients (4). One study reported mental disorders as concurrent NTM-related conditions (12). In our study, depression was also identified in NTM, which might be explained by the burden of COPD (13). These data suggest that an assessment of underlying disease is needed in managing NTM infection.

Our study had some limitations. First, because we used diagnosis codes, the distinction between pulmonary and other diseases is unclear, and we could not confirm whether the patients completely met the diagnostic criteria of NTM infection. Second, because we only have data for 2007-2016, we could not exclude patients who had received diagnoses before 2007 when estimating the annual incidence rate. Finally, our definition of NTM infection on the basis of the International Classification of Diseases code may have underestimated the true disease prevalence $(14,15)$ because prevalence could be affected by loss to follow-up or death during the study period.

In conclusion, we report a substantial increasing trend in the prevalence and incidence of NTM infection in South Korea during 2007-2016 and evaluated regional variations. These data facilitate a better understanding of the epidemiologic trends of NTM infection globally.

\section{Acknowledgments}

This work was supported by the National Research Foundation of Korea (NRF) Grant funded by the Ministry of Science and ICT (Information and Communication Technologies), South Korea (grant no. NRF2018R1C1B6001708; W. Myung), and by the Basic Science Research Program through the NRF of Korea funded by the Ministry of Education, South Korea (grant no. NRF-2018R1D1A1B07049034; H. Lee).

\section{About the Author}

Dr. Lee is a research assistant professor at the Institute of Health and Environment at Seoul National University, South Korea. Her work focuses on health impact assessment of environmental risk factors, including air pollutants and climatic factors.

\section{References}

1. Prevots DR, Marras TK. Epidemiology of human pulmonary infection with nontuberculous mycobacteria: a review. Clin Chest Med. 2015;36:13-34. http://dx.doi.org/10.1016/ j.ccm.2014.10.002

2. Ko RE, Moon SM, Ahn S, Jhun BW, Jeon K, Kwon OJ, et al. Changing epidemiology of nontuberculous mycobacterial lung diseases in a tertiary referral hospital in Korea between 2001 and 2015. J Korean Med Sci. 2018;33:e65. http://dx.doi.org/10.3346/ jkms.2018.33.e65

3. Cheol Seong S, Kim YY, Khang YH, Heon Park J, Kang HJ, Lee $\mathrm{H}$, et al. Data resource profile: the National Health Information 
Database of the National Health Insurance Service in South Korea Int J Epidemiol. 2017;46:799-800. http://dx.doi.org/10.1093/ije/ dyw 253

4. Ringshausen FC, Wagner D, de Roux A, Diel R, Hohmann D, Hickstein L, et al. Prevalence of nontuberculous mycobacterial pulmonary disease, Germany, 2009-2014. Emerg Infect Dis. 2016;22:1102-5. http://dx.doi.org/10.3201/ eid2206.151642

5. Adjemian J, Olivier KN, Seitz AE, Holland SM, Prevots DR Prevalence of nontuberculous mycobacterial lung disease in US Medicare beneficiaries. Am J Respir Crit Care Med. 2012;185:881-6. http://dx.doi.org/10.1164/rccm.201111-2016OC

6. Henkle E, Hedberg K, Schafer S, Novosad S, Winthrop KL. Population-based incidence of pulmonary nontuberculous mycobacterial disease in Oregon 2007 to 2012. Ann Am Thorac Soc. 2015;12:642-7. http://dx.doi.org/10.1513/ AnnalsATS.201412-559OC

7. Morimoto K, Iwai K, Uchimura K, Okumura M, Yoshiyama T, Yoshimori K, et al. A steady increase in nontuberculous mycobacteriosis mortality and estimated prevalence in Japan. Ann Am Thorac Soc. 2014;11:1-8. http://dx.doi.org/10.1513/ AnnalsATS.201303-067OC

8. Namkoong H, Kurashima A, Morimoto K, Hoshino Y, Hasegawa N, Ato M, et al. Epidemiology of pulmonary nontuberculous mycobacterial disease, Japan. Emerg Infect Dis. 2016;22:1116-7. http://dx.doi.org/10.3201/eid2206.151086

9. Adjemian J, Olivier KN, Seitz AE, Falkinham JO III, Holland SM, Prevots DR. Spatial clusters of nontuberculous mycobacterial lung disease in the United States. Am J Respir Crit Care Med. 2012;186:553-8. http://dx.doi.org/10.1164/ rccm.201205-0913OC

10. Adjemian J, Frankland TB, Daida YG, Honda JR, Olivier KN, Zelazny A, et al. Epidemiology of nontuberculous mycobacterial lung disease and tuberculosis, Hawaii, USA. Emerg Infect Dis. 2017;23:439-47. http://dx.doi.org/10.3201/ eid2303.161827

11. Olivier KN, Weber DJ, Wallace RJ Jr, Faiz AR, Lee JH, Zhang Y, et al.; Nontuberculous Mycobacteria in Cystic Fibrosis Study Group. Nontuberculous mycobacteria. I: multicenter prevalence study in cystic fibrosis. Am J Respir Crit Care Med. 2003;167:828-34. http://dx.doi.org/10.1164/ rccm.200207-6780C

12. Mirsaeidi M, Machado RF, Garcia JG, Schraufnagel DE. Nontuberculous mycobacterial disease mortality in the United States, 1999-2010: a population-based comparative study. PLoS One. 2014;9:e91879. http://dx.doi.org/10.1371/ journal.pone.0091879

13. Yohannes AM, Alexopoulos GS. Depression and anxiety in patients with COPD. Eur Respir Rev. 2014;23:345-9. http://dx.doi.org/10.1183/09059180.00007813

14. Prevots DR, Shaw PA, Strickland D, Jackson LA, Raebel MA, Blosky MA, et al. Nontuberculous mycobacterial lung disease prevalence at four integrated health care delivery systems. Am J Respir Crit Care Med. 2010;182:970-6. http://dx.doi.org/ 10.1164/rccm.201002-0310OC

15. Winthrop KL, Baxter R, Liu L, Varley CD, Curtis JR, Baddley JW, et al. Mycobacterial diseases and antitumour necrosis factor therapy in USA. Ann Rheum Dis. 2013;72:37-42. http://dx.doi.org/10.1136/annrheumdis-2011-200690

Address for correspondence: Byung Woo Jhun, Samsung Medical Center, Sungkyunkwan University School of Medicine, Division of Pulmonary and Critical Care Medicine, Department of Medicine, Irwon-ro 81, Gangnam-gu, Seoul 06351, South Korea; email: byungwoo.jhun@gmail.com

\section{EID Podcast: WWI and the 1918 Influenza Pandemic}

CDC's Dr. Terence Chorba

discusses his EID cover art essay about the 1918 influenza pandemic and the WWI painting by John Singer Sargent.

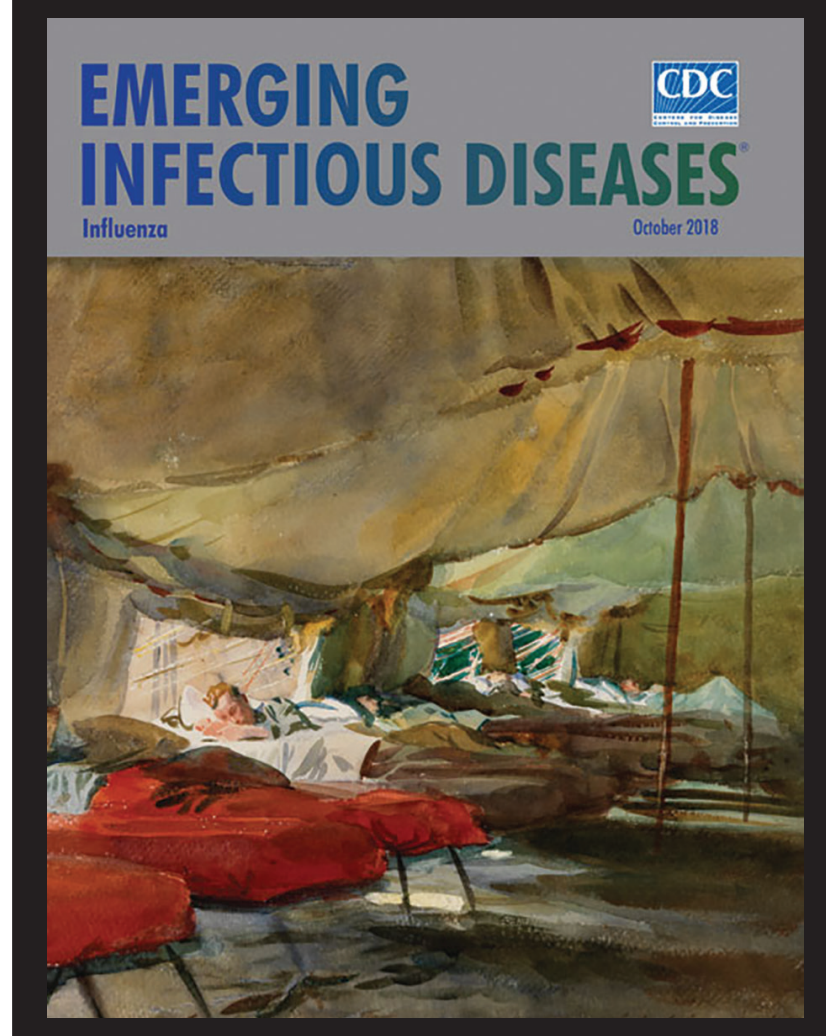

Visit our website to listen: https://tools.cdc.gov/medialibrary/ index.aspx\#/media/id/393699 